\title{
Solution of the equations of motion for a super non-Abelian sigma model in curved background by the super Poisson-Lie T-duality
}

\author{
Ali Eghbali \\ Department of Physics, Faculty of Basic Sciences, Azarbaijan Shahid Madani University, \\ 53714-161, Tabriz, Iran
}

E-mail: a.eghbali@azaruniv.edu

ABSTRACT: The equations of motion of a super non-Abelian T-dual sigma model on the Lie supergroup $\left(C_{1}^{1}+A\right)$ in the curved background are explicitly solved by the super Poisson-Lie T-duality. To find the solution of the flat model we use the transformation of supercoordinates, transforming the metric into a constant one, which is shown to be a supercanonical transformation. Then, using the super Poisson-Lie T-duality transformations and the dual decomposition of elements of Drinfel'd superdouble, the solution of the equations of motion for the dual sigma model is obtained. The general form of the dilaton fields satisfying the vanishing $\beta$-function equations of the sigma models is found. In this respect, conformal invariance of the sigma models built on the Drinfel'd superdouble $\left(\left(C_{1}^{1}+A\right), I_{(2 \mid 2)}\right)$ is guaranteed up to one-loop, at least.

KEywords: Conformal and W Symmetry, String Duality, Sigma Models

ARXiv EPRINT: 1409.3933 


\section{Contents}

1 Introduction 1

2 A review of the super Poisson-Lie symmetric sigma models on Lie supergroups

3 The super non-Abelian T-dual sigma models $\quad 7$

3.1 The original model $\quad 7$

3.2 The dual model 8

$\begin{array}{lll}4 & \text { Flat supercoordinates } & 9\end{array}$

5 Solving the equations for flat supercoordinates $\quad 10$

5.1 Supercoordinates transformation as a supercanonical transformation 11

6 Calculation of the dual solution using the super Poisson-Lie T-duality transformations

7 Invariance of the dilaton field under the supercoordinates transformation and conformal sigma models

8 Conclusion

\section{Introduction}

Non-linear sigma models with target space supermanifolds possess a number of interesting properties. They are of importance both in superstring theory [1] and condensed matter physics $[2,3]$. Perhaps the main motivation to study them comes from the AdS/CFT duality. Furthermore, it has been shown that sigma models on the coset supermanifolds can be used for quantizing superstring theory with Ramond-Ramond backgrounds. The well-known example is type IIB Green-Schwarz superstring considered on $A d S_{5} \times S^{5}$ background in terms of supercoset formalism [4] (see, e.g., [5]-[7]). In order to have a better understanding of how superstring theory might be quantized, some examples on the Lie supergroups $\operatorname{PSL}(n \mid n)$ were considered in [5, 6] and [8]. These Lie supergroups, having vanishing Killing form and Ricci-flat property, guarantee that the corresponding sigma models are conformally invariant up to one-loop [9]; this is a result of [6] in which these models are exactly conformal.

On the other hand, duality transformations have played an important role in string theory. Target space duality (T-duality) as a very important symmetry of string theories - or more generally two-dimensional sigma models - has been achieved in revealing the 
consequences of the isometry symmetry in string theory [10, 11]-[14]. In particular, it states the equivalence between string theories propagating on two different target spaces that contain some Abelian isometries [10,11]. Unlike the Abelian case [15], the nonAbelian T-duality transformation is not invertible since the isometry symmetries of the original theory are not preserved. So it was not possible to perform the inverse duality transformation to get back to the original model [16]. A solution to this problem was proposed by Klimčík and Ševera [17] where it was argued that the two models were dual in the sense of the so-called Poisson-Lie T-duality. The Poisson-Lie T-duality does not require the existence of isometry in the original target manifold. In this case, the model is not required to has an isometry symmetry, but there is still an action of a Lie group G on the target manifold and the Noetherian currents associated with this action are required to be integrable, that is, they satisfy Maurer-Cartan equations with group structure of $\tilde{G}$ (with the same dimension of $G$ ) so that $G$ and $\tilde{G}$ have Poisson-Lie structure and their Lie algebras are dual to each other in the sense that they define a bialgebra structure [18].

The solution of the sigma models in time-dependent and curved backgrounds is often very complicated. In ref. [17] the procedure for transforming the solutions of a sigma model to those of a dual one has been described. Afterwards, this procedure was extended in such a way that by use of the transformation of group coordinates of the flat model to those for which the metric is constant, a classical solution of the equations of motion for a sigma model in curved background was found in [19] (see, also, [20]). Moreover, prior to the procedures done in [19] and [20], conformally invariant three-dimensional sigma models on solvable Lie groups, which were Poisson-Lie T-dual or plural to sigma models in the flat background with the constant dilaton, were investigated in [21] (see, also, [22, 23]).

We have recently generalized Piosson-Lie symmetry on manifolds to supermanifolds and have constructed super Poisson-Lie T-dual sigma models on Lie supergroups [24], specially on supermanifolds [25]. Furthermore, we have shown that the super Poisson-Lie T-duality relates the WZW models based on the Lie supergroups to each other [26] (see, also, [27] ). In this paper, we are going to solve the equations of motion of a super nonAbelian T-dual sigma model in curved background by the super Poisson-Lie T-duality. To this end, we use the fact that these models in curved background can be transformed into the flat ones. By solving partial differential equations that follow the transformation properties of the connection components we find the transformation between supergroup coordinates of the model in curved background and Riemannian coordinates (flat supercoordinates) for which the metric is constant. The model is built on the Lie supergroup $\left(C_{1}^{1}+A\right)$. The Lie superalgebra $\left(\mathcal{C}_{1}^{1}+\mathcal{A}\right)[28]$ of the Lie supergroup $\left(C_{1}^{1}+A\right)$ is a $(2 \mid 2)$ dimensional Lie superalgebra with two bosonic and two fermionic generators. Because of performability of the super Poisson-Lie T-duality transformations, namely finding coordinates of the dual decompositions which depend on the complexity of the structure of Drinfel'd superdouble, we have chosen one of the Lie sub-supergroups of the decomposition of the Drinfel'd superdouble to be Abelian Lie supergroup. In this case, the super Poisson-Lie T-duality reduces to the super non-Abelian T-duality. By reducing flat supercoordinates to the solution of the wave equation we obtain solution of the equations of motion of the original model. We, furthermore, show that the transformation of the flat supercoordinates is a 
supercanonical transformation. In the following, by using the super Poisson-Lie T-duality transformation that follows two possible decomposition of elements of the Drinfel'd superdouble, we get the solution of the equations of motion for the dual model. The reason why it is very desirable to find the transformation between the supergroup coordinates and the flat supercoordinates is that in the flat supercoordinates the metric becomes constant. Therefore, the vanishing $\beta$-function equations become very simple. On the other hand, finding solution for conformally invariant sigma models in curved backgrounds is generally a difficult problem. Thus, with regard to the above discussion we easily solve equations for the dilaton field of the flat sigma model in the flat supercoordinates. Then, to get the general dilaton of the original sigma model which satisfies the vanishing $\beta$-function equations in curved background, we use the flat supercoordinates transformation. Finally, we obtain the general form of the dilaton field satisfying the vanishing $\beta$-function equations for the dual model in terms of the coordinates of the dual Lie supergroup.

The plan of the paper is as follows. In section 2, we briefly review the super Poisson-Lie symmetry and mutual super Poisson-Lie T-dual sigma models on Lie supergroups based on our previous papers. In section 3 , we introduce the Lie superalgebra $\left(\mathcal{C}_{1}^{1}+\mathcal{A}\right)$ and the Lie superalgebra of Drinfel'd superdouble $\left(\left(\mathcal{C}_{1}^{1}+\mathcal{A}\right), \mathcal{I}_{(2 \mid 2)}\right)$. Moreover, in section 3, the $(2 \mid 2)$ dimensional super non-Abelian T-dual sigma models based on the Drinfel'd superdouble $\left(\left(C_{1}^{1}+A\right), I_{(2 \mid 2)}\right)$ are constructed. Section 4 is devoted to the presentation of a method for finding flat supercoordinates. In section 5 we find the transformation of supercoordinates which makes the metric constant. Also in section 5 we prove that the transformation of the flat supercoordinates is indeed a supercanonical transformation. In section 6 , utilizing the solution of the wave equation and using the super Poisson-Lie T-duality transformations we get the solution of the equations of motion for the dual sigma model. Finding the general form of the dilaton fields satisfying the vanishing $\beta$-function equations of the sigma models is given in section 7. Finally, we present our conclusions.

\section{A review of the super Poisson-Lie symmetric sigma models on Lie supergroups}

To set our notation, let us briefly review the construction of the super Poisson-Lie T-dual sigma models by means of Drinfel'd superdoubles. Consider a two-dimensional sigma model action on a supermanifold $M$ as ${ }^{1}$

$$
S=\frac{1}{2} \int_{\Sigma} d \sigma^{+} d \sigma^{-}(-1)^{|\Upsilon|} \partial_{+} \Phi^{\Upsilon} \mathcal{E}_{\Upsilon \Lambda}(\Phi) \partial_{-} \Phi^{\Lambda}
$$

where $\Sigma$ is the worldsheet and $\partial_{ \pm}$are the derivatives with respect to the standard lightcone variables $\sigma^{ \pm}:=\frac{1}{2}(\tau \pm \sigma)$ on the worldsheet, and $\mathcal{E}_{\Upsilon \Lambda}=G_{\Upsilon \Lambda}+B_{\Upsilon \Lambda} \cdot{ }^{2}$ The coordinates

\footnotetext{
${ }^{1}$ We note that $|\Upsilon|$ denotes the parity of $\Upsilon$ where $|\Upsilon|=0$ for the bosonic coordinates and $|\Upsilon|=1$ for the fermionic coordinates; here and in the following we use the notation [29], e.g., $(-1)^{|\Upsilon|}:=(-1)^{\Upsilon}$.

${ }^{2}$ Note that $G_{\Upsilon \Lambda}$ and $B_{\Upsilon \Lambda}$ are the components of the supersymmetric metric $G$ and the antisupersymmetric tensor field $B$, respectively,$$
G_{\Upsilon \Lambda}=(-1)^{\Upsilon \Lambda} G_{\Lambda \Upsilon}, \quad B_{\Upsilon \Lambda}=-(-1)^{\Upsilon \Lambda} B_{\Lambda \Upsilon} .
$$

We will assume that the metric ${ }_{\Upsilon} G_{\Lambda}$ is superinvertible and its superinverse is shown by $G^{\Upsilon \Lambda}$.
} 
$\Phi^{\Upsilon}$ include the bosonic and the fermionic coordinates, and the label $\Upsilon$ runs over $\mu=$ $0, \cdots, d_{B}-1$ and $\alpha=1, \cdots, d_{F}$ where $d_{B}$ and $d_{F}$ indicate the dimension of the bosonic coordinates and the fermionic coordinates, respectively. Thus, the superdimension of the supermanifold is written as $\left(d_{B} \mid d_{F}\right)$. We define, respectively, the Christoffel symbols (the components of the connection) and the torsion $\operatorname{as}^{3}$

$$
\begin{aligned}
\Gamma_{\Lambda \Xi}^{\Upsilon} & =\frac{(-1)^{\Delta}}{2} G^{\Upsilon \Delta}\left[\frac{G_{\Delta \Lambda} \overleftarrow{\partial}}{\partial \Phi^{\Xi}}+(-1)^{\Lambda \Xi} \frac{G_{\Delta \Xi} \overleftarrow{\partial}}{\partial \Phi^{\Lambda}}-(-1)^{\Delta(\Lambda+\Xi)} \frac{G_{\Lambda \Xi} \overleftarrow{\partial}}{\partial \Phi^{\Delta}}\right] \\
H_{\Upsilon \Xi}^{\Lambda} & =\frac{(-1)^{\Delta}}{2} G^{\Lambda \Delta}\left[\frac{B_{\Delta \Upsilon} \overleftarrow{\partial}}{\partial \Phi^{\Xi}}+(-1)^{\Xi(\Upsilon+\Delta)} \frac{B_{\Xi \Delta \Delta} \overleftarrow{\partial}}{\partial \Phi^{\Upsilon}}+(-1)^{\Delta(\Upsilon+\Xi)} \frac{B_{\Upsilon \Xi} \overleftarrow{\partial}}{\partial \Phi^{\Delta}}\right]
\end{aligned}
$$

Thus, one gets the equations of motion for the action (2.1) as

$$
\partial_{+} \partial_{-} \Phi^{\Upsilon}+\left(\Gamma_{\Lambda \Xi}^{\Upsilon}-H_{\Lambda \Xi}^{\Upsilon}\right) \partial_{-} \Phi^{\Xi} \partial_{+} \Phi^{\Lambda}=0 .
$$

If the Noether's current one-forms corresponding to the right action of the Lie supergroup $G$ on the target space $M$ are not closed and satisfy the Maurer-Cartan equation [29] on the extremal surfaces, we say that the sigma model (2.1) has the super Poisson-Lie symmetry with respect to the Lie supergroup $\tilde{G}$ (the dual Lie supergroup to $G$ ) [24]. It is a condition that is given by the following relation $[24]^{4}$

$$
\mathcal{L}_{V_{i}}\left(\mathcal{E}_{\Upsilon \Lambda}\right)=(-1)^{i(\Upsilon+k)} \mathcal{E}_{\Upsilon \Xi}\left(V^{s t}\right)^{\Xi}{ }_{k}\left(\tilde{\mathcal{Y}}_{i}\right)^{k j}{ }_{j} V^{\Omega}{ }_{\Omega} \mathcal{E}_{\Lambda},
$$

where $\mathcal{L}_{V_{i}}$ stands for the Lie derivative corresponding to the left invariant supervector fields $V_{i}$ (defined with left derivative) $[24]$ and $\left(\tilde{\mathcal{Y}}_{i}\right)^{j k}=-\tilde{f}^{j k}{ }_{i}$ are the adjoint representations of Lie superalgebra $\tilde{\mathcal{G}}$ (the dual Lie superalgebra to $\mathcal{G}$ ). When $\mathcal{L}_{V_{i}}\left(\mathcal{E}_{\Upsilon \Lambda}\right)=0$, the super Poisson-Lie T-duality is replaced by the super non-Abelian T-duality, i.e., the Lie supergroup $G$ is a isometry supergroup of $M$ and we have the conserved currents.

Let us consider the dualizable sigma models defined on the Lie supergroup space (the generalization to the case when a Lie supergroup $G$ acts freely on the target space). The super Poisson-Lie dualizable sigma models can be formulated on a Drinfel'd superdouble $D \equiv(G, \tilde{G})[30]$, a Lie supergroup whose $\mathcal{D}$ admits a decomposition $\mathcal{D}=\mathcal{G} \oplus \tilde{\mathcal{G}}$ into a pair of sub-superalgebras maximally isotropic with respect to a supersymmetric and ad-invariant non-degenerate bilinear form $<., .>$ which is defined by the brackets

$$
0=<X_{i}, X_{j}>=<\tilde{X}^{i}, \tilde{X}^{j}>, \quad \delta^{i}{ }_{j}=<\tilde{X}^{i}, X_{j}>=(-1)^{i j}<X_{j}, \tilde{X}^{i}>.
$$

\footnotetext{
${ }^{3}$ Notation: If $f$ be a differentiable function on $\mathbf{R}_{c}^{m} \times \mathbf{R}_{a}^{n}\left(\mathbf{R}_{c}^{m}\right.$ are subset of all real numbers with dimension $m$ while $\mathbf{R}_{a}^{n}$ are subset of all odd Grassmann variables with dimension $n$ ), then, the relation between the left partial differentiation and right one is given by

$$
\Upsilon \vec{\partial} f:=\frac{\vec{\partial} f}{\partial \Phi^{\Upsilon}}=(-1)^{\Upsilon(|f|+1)} \frac{f \overleftarrow{\partial}}{\partial \Phi^{\Upsilon}}
$$

where $|f|$ indicates the grading of $f[29]$.

${ }^{4}$ The superscript "st" means supertransposition of the matrix [29].
} 
Such decomposition is called Manin supertriple $(\mathcal{D}, \mathcal{G}, \tilde{\mathcal{G}})$. The generators of $\mathcal{G}$ and $\tilde{\mathcal{G}}$ are, respectively, denoted to $X_{i}$ and $\tilde{X}^{i}$ and satisfy the (anti)commutation relations [31]

$$
\begin{array}{lr}
{\left[X_{i}, X_{j}\right]=f^{k}{ }_{i j} X_{k}, \quad\left[\tilde{X}^{i}, \tilde{X}^{j}\right]=\tilde{f}_{k}{ }_{k} \tilde{X}^{k},} \\
{\left[X_{i}, \tilde{X}^{j}\right]=(-1)^{j} \tilde{f}^{j k}{ }_{i} X_{k}+(-1)^{i} f^{j}{ }_{k i} \tilde{X}^{k} .}
\end{array}
$$

The structure constants $f^{k}{ }_{i j}$ and $\tilde{f}_{k}^{i j}$ are subject to the mixed super Jacobi identities [31]

$$
f_{j k}^{m} \tilde{f}_{m}^{i l}=f_{m k}^{i} \tilde{f}_{j}^{m l}+f_{j m}^{l} \tilde{f}_{k}^{i m}+(-1)^{j l} f_{j m}^{i} \tilde{f}_{k}^{m l}+(-1)^{i k} f_{m k}^{l} \tilde{f}_{j}^{i m} .
$$

Now, we assume $\varepsilon^{+}$is a $\left(d_{B} \mid d_{F}\right)$-dimensional linear sub-superspace and $\varepsilon^{-}$is its orthogonal complement so that $\varepsilon^{+}+\varepsilon^{-}$span the whole Lie superalgebra $\mathcal{D}$. To determine a dual pair of the sigma models with the targets $G$ and $\tilde{G}$, one can consider the following equation of motion for the mapping $l\left(\sigma^{+}, \sigma^{-}\right)$from the worldsheet into the Drinfel'd superdouble $D,^{5}$

$$
<\partial_{ \pm} l l^{-1}, \varepsilon^{\mp}>=0
$$

Then, using equation (2.9) and the decomposition of an arbitrary element of $D$ in the vicinity of the unit element of $D$ as

$$
l\left(\sigma^{+}, \sigma^{-}\right)=g\left(\sigma^{+}, \sigma^{-}\right) \tilde{h}\left(\sigma^{+}, \sigma^{-}\right), \quad g \in G, \quad \tilde{h} \in \tilde{G},
$$

we obtain

$$
<g^{-1} \partial_{ \pm} g+\partial_{ \pm} \tilde{h} \tilde{h}^{-1}, g^{-1} \varepsilon^{\mp} g>=0
$$

for which

$$
g^{-1} \varepsilon^{ \pm} g=\operatorname{Span}\left\{X_{i} \pm E_{i j}^{ \pm}(g) \tilde{X}^{j}\right\},
$$

where $E^{ \pm}: \mathcal{G} \rightarrow \tilde{\mathcal{G}}$ is a non-degenerate linear map, and $E_{i j}^{-}=\left(E_{i j}^{+}\right)^{s t}=(-1)^{i j} E_{j i}^{+}$. Now, inserting relation (2.12) into (2.11) and using relation (2.6), we can write the field equation (2.11) in the form [24]

$$
\begin{aligned}
\left(\partial_{+} \tilde{h} \tilde{h}^{-1}\right)_{i} & =(-1)^{j}\left(g^{-1} \partial_{+} g\right)^{j} E_{j i}^{+}(g), \\
\left(\partial_{-} \tilde{h} \tilde{h}^{-1}\right)_{i} & =-E_{i j}^{+}(g)\left(g^{-1} \partial_{-} g\right)^{j},
\end{aligned}
$$

where $\left(\partial_{ \pm} \tilde{h} \tilde{h}^{-1}\right)_{i}$ are right invariant one-forms on $\tilde{G}$ and satisfy in the following super flat connection equations

$$
\partial_{+}\left(\partial_{-} \tilde{h} \tilde{h}^{-1}\right)_{i}-\partial_{-}\left(\partial_{+} \tilde{h} \tilde{h}^{-1}\right)_{i}-(-1)^{j k} \tilde{f}_{i}^{j k}\left(\partial_{-} \tilde{h} \tilde{h}^{-1}\right)_{j}\left(\partial_{+} \tilde{h} \tilde{h}^{-1}\right)_{k}=0 .
$$

However, one can show that equations (2.15) are the field equations of the sigma model with the action

$$
S=\frac{1}{2} \int d \sigma^{+} d \sigma^{-}(-1)^{i}\left(g^{-1} \partial_{+} g\right)^{i} E_{i j}^{+}(g)\left(g^{-1} \partial_{-} g\right)^{j},
$$

\footnotetext{
${ }^{5}$ Note that equation (2.9) is the field equation coming from the first-order Hamiltonian action for field configurations $l(\tau, \sigma) \in D \quad[32,33]$.
} 
in which

$$
\left(g^{-1} \partial_{ \pm} g\right)^{i}:=L_{ \pm}^{(l)^{i}}=\partial_{ \pm} \Phi_{\Upsilon}^{\Upsilon} L^{(l)^{i}}
$$

are left invariant one-forms with left derivative. Comparing the action (2.16) with the action (2.1) we obtain

$$
\mathcal{E}_{\Upsilon \Lambda}=(-1)^{i} L_{\Upsilon}^{(l)}{ }^{i} E_{i j}^{+}(g)\left(L^{(l)^{s t}}\right)^{j} \Lambda .
$$

Then, using the fact that left invariant one-forms are dual to the supervector fields, i.e., ${ }_{i} V_{\Upsilon}^{\Upsilon} L^{(l)^{j}}={ }_{i} \delta^{j}$, one can rewrite the equations (2.13) and (2.14) in the following form

$$
\begin{aligned}
& \left(\partial_{+} \tilde{h} \tilde{h}^{-1}\right)_{i}=(-1)^{\Upsilon} \partial_{+} \Phi^{\Upsilon} \mathcal{E}_{\Upsilon \Lambda}\left(V^{s t}\right)^{\Lambda}, \\
& \left(\partial_{-} \tilde{h} \tilde{h}^{-1}\right)_{i}=-(-1)^{i+\Upsilon}{ }_{i} V^{\Upsilon} \mathcal{E}_{\Upsilon \Lambda} \partial_{-} \Phi^{\Lambda} .
\end{aligned}
$$

Also, one can rewrite the action (2.16) in the following form

$$
S=\frac{1}{2} \int d \sigma^{+} d \sigma^{-}(-1)^{i} R_{+}^{(l)^{i}} F_{i j}^{+}(g) R_{-}^{(l)^{j}}
$$

where $R_{ \pm}(l)^{i}$ 's are right invariant one-forms with left derivative and are defined by

$$
R_{\alpha}^{(l)^{i}}=<\partial_{\alpha} g g^{-1}, \tilde{X}^{i}>
$$

The matrix $F_{i j}^{+}(g)$ of the sigma model is of the form

$$
F^{+}(g)=\left(\Pi(g)+\left(E_{0}^{+}\right)^{-1}(e)\right)^{-1}, \quad \Pi^{i j}(g)=(-1)^{k} b^{i k}(g)\left(a^{-1}\right)_{k}^{j}(g),
$$

where $E_{0}^{+}(e)$ is a constant matrix and $\Pi(g)$ defines the super Poisson structure on the Lie supergroup $G$, and the sub-matrices $a(g)$ and $b(g)$ are constructed in terms of the bilinear forms as

$$
a_{i}^{j}(g)=<g^{-1} X_{i} g, \tilde{X}^{j}>, \quad b^{i j}(g)=<g^{-1} \tilde{X}^{i} g, \tilde{X}^{j}>.
$$

Equivalently, by using the decomposition

$$
l\left(\sigma^{+}, \sigma^{-}\right)=\tilde{g}\left(\sigma^{+}, \sigma^{-}\right) h\left(\sigma^{+}, \sigma^{-}\right), \quad \tilde{g} \in \tilde{G}, \quad h \in G,
$$

and by exchanging $G \leftrightarrow \tilde{G}, \mathcal{G} \leftrightarrow \tilde{\mathcal{G}}$ and ${ }_{i} E_{0_{j}}^{+}(e) \leftrightarrow \tilde{E}_{0}^{+i j}(\tilde{e})=\left(E_{0}^{+}\right)^{-1^{i j}}(e)$, the dual sigma model is obtained. Furthermore, we note that equations (2.10), (2.13), (2.14) and (2.25) define the so-called super Poisson-Lie T-duality transformations.

Notice that if we take a dual Abelian Lie supergroup $\left(\tilde{f}_{k}^{i j}=0\right)$ with local coordinates $\tilde{\Phi}_{k}$ characterizing the supergroup element $\tilde{g}$, then, in this case we have

$$
\Pi^{i j}(g)=0, \quad \tilde{\Pi}^{i j}(\tilde{g})=-\tilde{\Phi}_{k} f^{k}{ }_{i j},
$$

recovering, thus, the super non-Abelian T-duality. To continue, we shall present an example of (2|2)-dimensional super non-Abelian T-dual sigma models with a curved background for which the sigma model can be explicitly solved by the super Poisson-Lie T-duality transformations. This model is obtained from the Drinfel'd superdouble $\left(\left(C_{1}^{1}+A\right), I_{(2 \mid 2)}\right)$. 


\section{The super non-Abelian T-dual sigma models}

Both the original and the dual geometries of the super Poisson-Lie T-dualizable sigma models are derived from the so-called Drinfel'd superdouble which is a Lie supergroup. We shall construct a dualizable sigma model on the (2|2)-dimensional Lie supergroup $\left(C_{1}^{1}+A\right)$ when the dual Lie supergroup is the (2|2)-dimensional Abelian Lie supergroup, hence, the super Poisson-Lie T-duality reduces to the super non-Abelian T-duality. To this end, we first introduce the Lie superalgebra $\left(\mathcal{C}_{1}^{1}+\mathcal{A}\right)$ [28] of the Lie supergroup $\left(C_{1}^{1}+A\right)$. As mentioned in introduction section, the Lie superalgebra $\left(\mathcal{C}_{1}^{1}+\mathcal{A}\right)$ possesses two bosonic generators $X_{1}$ and $X_{2}$ along with two fermionic ones $X_{3}$ and $X_{4}$. These four generators obey the following set of non-trivial (anti)commutation relations [28]

$$
\left[X_{1}, X_{2}\right]=X_{2}, \quad\left[X_{1}, X_{3}\right]=X_{3}, \quad\left\{X_{3}, X_{4}\right\}=X_{2} .
$$

The Lie superalgebra of the Drinfel'd superdouble which we refer to as $\left(\left(\mathcal{C}_{1}^{1}+\mathcal{A}\right), \mathcal{I}_{(2 \mid 2)}\right)$ is denoted by generators $\left\{X_{1}, X_{2}, \tilde{X}^{1}, \tilde{X}^{2} ; X_{3}, X_{4}, \tilde{X}^{3}, \tilde{X}^{4}\right\}$ and the following nonzero (anti)commutation relations

$$
\begin{array}{llrl}
{\left[X_{1}, X_{2}\right]} & =X_{2}, & {\left[X_{1}, X_{3}\right]=X_{3},} & \left\{X_{3}, X_{4}\right\}=X_{2}, \\
{\left[X_{1}, \tilde{X}^{2}\right]=-\tilde{X}^{2},} & {\left[X_{1}, \tilde{X}^{3}\right]=-\tilde{X}^{3},} & {\left[X_{2}, \tilde{X}^{2}\right]=\tilde{X}^{1},} \\
{\left[X_{3}, \tilde{X}^{2}\right]=-\tilde{X}^{4},} & {\left[X_{4}, \tilde{X}^{2}\right]=-\tilde{X}^{3},} & \left\{X_{3}, \tilde{X}^{3}\right\}=-\tilde{X}^{1},
\end{array}
$$

where $\left\{\tilde{X}^{1}, \tilde{X}^{2}\right\}$ and $\left\{\tilde{X}^{3}, \tilde{X}^{4}\right\}$ are the respective bosonic and fermionic generators of the Abelian Lie superalgebra.

\subsection{The original model}

The super Poisson-Lie T-dual sigma models are usually expressed in terms of supergroup coordinates. These coordinates are given by the Lie supergroup structure and follow from the possibility to express the elements of the Lie supergroup as a product of elements of one-parametric sub-(super)groups. In order to construct the corresponding original sigma model with the Lie supergroup $\left(C_{1}^{1}+A\right)$ as the target space, we use the supergroup coordinates $\Phi^{\Upsilon}=\{x, y ; \psi, \chi\}$, for which the elements of the Lie supergroup $G$ are parametrized as

$$
g=e^{\chi X_{4}} e^{y X_{2}} e^{x X_{1}} e^{\psi X_{3}}
$$

where we have, here, assumed that $g$ is a mapping from $\Sigma$ into $G$. The fields $x(\tau, \sigma)$ and $y(\tau, \sigma)$ are bosonic while $\psi(\tau, \sigma)$ and $\chi(\tau, \sigma)$ are fermoinic fields. Inserting the above parametrization in $(2.22)$ and $(2.17), R_{ \pm}^{(l)}{ }^{i}$ 's and $L_{ \pm}^{(l)}{ }^{i}$ 's are, respectively, found to be

$$
\begin{array}{ll}
R_{ \pm}^{(l)^{X_{1}}}=\partial_{ \pm} x, & R_{ \pm}^{(l)}{ }^{X_{2}}=-\partial_{ \pm} x y+\partial_{ \pm} y+\partial_{ \pm} \psi e^{x} \chi, \\
\left.R_{ \pm}^{(l)}\right)^{X_{3}}=-\partial_{ \pm} \psi e^{x}, & R_{ \pm}^{(l)^{X_{4}}}=-\partial_{ \pm} \chi,
\end{array}
$$

and

$$
\begin{array}{ll}
L_{ \pm}^{(l)^{X_{1}}}=\partial_{ \pm} x, & L_{ \pm}^{(l)}{ }^{X_{2}}=\partial_{ \pm} y e^{-x}-\partial_{ \pm} \chi \psi, \\
L_{ \pm}^{(l)^{X_{3}}}=-\partial_{ \pm} x \psi-\partial_{ \pm} \psi, & L_{ \pm}^{(l)^{X_{4}}}=-\partial_{ \pm} \chi .
\end{array}
$$


In this case, $\Pi(g)=0$ because the dual Lie supergroup is Abelin. Hence, choosing the sigma model matrix $E_{0}^{+}(e)$ at the unit element of the Lie supergroup $\left(C_{1}^{1}+A\right)$ as

$$
E_{0_{i j}}^{+}(e)=\left(\begin{array}{cccc}
0 & K & 0 & 0 \\
K & 0 & 0 & 0 \\
0 & 0 & 0 & K \\
0 & 0 & -K & 0
\end{array}\right),
$$

where $K$ is a non-zero constant, and using the first equation of (2.23), the original sigma model action is worked out as follows:

$$
\begin{aligned}
S= & \frac{K}{2} \int d \sigma^{+} d \sigma^{-}\left(-2 y \partial_{+} x \partial_{-} x+\partial_{+} x \partial_{-} y+\partial_{+} y \partial_{-} x\right. \\
& \left.-\partial_{+} x e^{x} \chi \partial_{-} \psi+\partial_{+} \psi e^{x} \chi \partial_{-} x-\partial_{+} \psi e^{x} \partial_{-} \chi+\partial_{+} \chi e^{x} \partial_{-} \psi\right) .
\end{aligned}
$$

This model is not only Ricci flat and torsionless but also is flat in the sense that its Riemann tensor vanishes. Thus, we shall deal with a model having $\mathcal{E}_{\Upsilon_{\Lambda}}=G_{\Upsilon \Lambda}$ so that

$$
G_{\Upsilon \Lambda}(\Phi)=\left(\begin{array}{cccc}
-2 K y & K & -K e^{x} \chi & 0 \\
K & 0 & 0 & 0 \\
-K e^{x} \chi & 0 & 0 & K e^{x} \\
0 & 0 & -K e^{x} & 0
\end{array}\right)
$$

\subsection{The dual model}

In the same way to construct the dual sigma model, we parametrize the corresponding Lie supergroup (Abelian Lie supergroup) with bosonic coordinates $\{\tilde{x}, \tilde{y}\}$ and fermionic ones $\{\tilde{\psi}, \tilde{\chi}\}$ so that its elements can be written as:

$$
\tilde{g}=e^{\tilde{\chi} \tilde{X}^{4}} e^{\tilde{y} \tilde{X}^{2}} e^{\tilde{x} \tilde{X}^{1}} e^{\tilde{\psi} \tilde{X}^{3}} .
$$

Since the dual Lie supergroup is Abelian, so the components of the right invariant oneforms are simply calculated to be ${ }_{\Upsilon} \tilde{R}_{ \pm i}^{(l)}={ }_{\Upsilon} \tilde{\delta}_{i}\left({ }_{\Upsilon} \tilde{\delta}_{i}\right.$ is the ordinary delta function). Using the structure constants of the Lie superalgebra $\left(\mathcal{C}_{1}^{1}+\mathcal{A}\right)$ (relation $(3.1)$ ) and then utilizing the second equation of (2.26), the super Poisson structure on the dual Lie supergroup is found to be

$$
\tilde{\Pi}_{i j}(\tilde{g})=\left(\begin{array}{cccc}
0 & -\tilde{y} & -\tilde{\psi} & 0 \\
\tilde{y} & 0 & 0 & 0 \\
\tilde{\psi} & 0 & 0 & -\tilde{y} \\
0 & 0 & -\tilde{y} & 0
\end{array}\right) .
$$

It is quite interesting that the super Poisson structure is superinvertible, because one can use the relation

$$
\tilde{\omega}^{\Upsilon \Lambda}(\tilde{g})=(-1)^{i}\left(\tilde{L}^{(l)^{-s t}}\right)^{\Upsilon i} \tilde{\Pi}_{i j}(\tilde{g})\left(\tilde{L}^{(l)^{-1}}\right)^{j \Lambda},
$$


to construct a even supersymplectic two-form ${ }^{6} \tilde{\omega}$ on the dual supergroup supermanifold $\tilde{G}=I_{(2 \mid 2)}$ as follows:

$$
\tilde{\omega}=\frac{1}{\tilde{y}}\left(d \tilde{x} \wedge d \tilde{y}+\frac{\tilde{\psi}}{\tilde{y}} d \tilde{y} \wedge d \tilde{\chi}-d \tilde{\psi} \wedge d \tilde{\chi}\right) .
$$

It is easy to show that $d \tilde{\omega}=0$. Moreover, one can write the $\tilde{\omega}$ as the exterior derivative of one-form

$$
\tilde{\eta}=f(\tilde{x}) d \tilde{x}+\frac{\tilde{x}}{\tilde{y}} d \tilde{y}+\mathcal{C} \tilde{\psi} d \tilde{\psi}-\frac{\tilde{\psi}}{\tilde{y}} d \tilde{\chi}
$$

where $f(\tilde{x})$ is an arbitrary function of $\tilde{x}$ and $\mathcal{C}$ is an even, real constant. Let us, now, write the action of dual sigma model. Substituting (3.10) in the first equation of (2.23) (equation (2.23) in the dual form) and then using the fact that $\left(\tilde{E}_{0}^{+}\right)^{-1}(\tilde{e})=E_{0}^{+}(e)$, the dual sigma model action is obtained to be

$$
\begin{aligned}
\tilde{S}= & \frac{1}{2} \int d \sigma^{+} d \sigma^{-}\left(\frac{1}{K^{2}-\tilde{y}^{2}}\right)\left[(K-\tilde{y})\left(\partial_{+} \tilde{x} \partial_{-} \tilde{y}-\partial_{+} \tilde{\psi} \partial_{-} \tilde{\chi}\right)\right. \\
& \left.+(K+\tilde{y})\left(\partial_{+} \tilde{y} \partial_{-} \tilde{x}+\partial_{+} \tilde{\chi} \partial_{-} \tilde{\psi}\right)-\partial_{+} \tilde{y} \tilde{\psi} \partial_{-} \tilde{\chi}-\partial_{+} \tilde{\chi} \tilde{\psi} \partial_{-} \tilde{y}\right] .
\end{aligned}
$$

Comparing the above action with the sigma model action (2.1), we can elicit the metric $\tilde{G}_{\Upsilon \Lambda}$ and the field $\tilde{B}_{\Upsilon \Lambda}$ as

$$
\tilde{G}_{\Upsilon \Lambda}=\frac{K}{K^{2}-\tilde{y}^{2}}\left(\begin{array}{cccc}
0 & 1 & 0 & 0 \\
1 & 0 & 0 & 0 \\
0 & 0 & 0 & 1 \\
0 & 0 & -1 & 0
\end{array}\right), \quad \tilde{B}_{\Upsilon \Lambda}=\frac{1}{K^{2}-\tilde{y}^{2}}\left(\begin{array}{cccc}
0 & -\tilde{y} & 0 & 0 \\
\tilde{y} & 0 & 0 & -\tilde{\psi} \\
0 & 0 & 0 & -\tilde{y} \\
0 & \tilde{\psi} & -\tilde{y} & 0
\end{array}\right) .
$$

The investigated dual model has non-zero anti-supersymmetric part $\tilde{B}_{\Upsilon \Lambda}$ of the tensor $\tilde{\mathcal{E}}_{\Upsilon \Lambda}$, but it is flat in the sense that its scalar curvature vanishes.

\section{Flat supercoordinates}

Riemannian coordinates on supermanifolds are one type of coordinates where the sigma models live. In these coordinates, the metric on supermanifold have a special simple form. The Riemannian coordinates of the flat metrics are, here, called flat supercoordinates.

We know that the components of the connection do not transform as the components of a mixed tensor field. Thus, when the transform is from one coordinate basis to another, then, $\Gamma_{\Lambda \Delta}^{\Upsilon}$ transforms as follows [29]:

$$
\Gamma_{\Lambda \Delta}^{\Upsilon}=(-1)^{C(B+\Lambda)}\left(\frac{\Phi^{\Upsilon \overleftarrow{\partial}}}{\partial \Omega^{A}}\right) \bar{\Gamma}_{B C}^{A}\left(\frac{\Omega^{B} \overleftarrow{\partial}}{\partial \Phi^{\Lambda}}\right)\left(\frac{\Omega^{C} \overleftarrow{\partial}}{\partial \Phi^{\Delta}}\right)+\left(\frac{\Phi^{\Upsilon \overleftarrow{\partial}}}{\partial \Omega^{A}}\right)\left(\left(\frac{\Omega^{A} \overleftarrow{\partial}}{\partial \Phi^{\Lambda}}\right) \frac{\overleftarrow{\partial}}{\partial \Phi^{\Delta}}\right)
$$

\footnotetext{
${ }^{6}$ Note that in terms of the coordinate basis $\left\{d \tilde{\Phi}^{\Lambda}\right\}$, a two-form $\tilde{\omega}$ is defined as $\tilde{\omega}=\frac{(-1)^{\Upsilon \Lambda}}{2} \tilde{\omega}_{\Upsilon \Lambda} d \tilde{\Phi}^{\Upsilon} \wedge d \tilde{\Phi}^{\Lambda}$ (for more detailed description see DeWITT's book [29]).
} 
For finding the flat supercoordinates $\Omega^{A}$ (here, $\Omega^{A}=\left\{\Omega^{1}, \Omega^{2} ; \Omega^{3}, \Omega^{4}\right\}$, where $\Omega^{1}$ and $\Omega^{2}$ are bosonic coordinates while $\Omega^{3}$ and $\Omega^{4}$ are fermionic ones), we shall use formula (4.1). In these supercoordinates the metric becomes constant and, therefore, the elements of $\bar{\Gamma}_{B C}^{A}$ vanish. Then, we get the following system of partial differential equations (PDEs) for $\Omega(\Phi)$

$$
(-1)^{\Upsilon+\Upsilon B} \frac{\vec{\partial} \Omega^{B}}{\partial \Phi^{\Upsilon}} \Gamma_{\Lambda \Delta}^{\Upsilon}=(-1)^{(\Lambda+\Delta)(1+B)} \frac{\vec{\partial}}{\partial \Phi^{\Lambda}}\left(\frac{\vec{\partial} \Omega^{B}}{\partial \Phi^{\Delta}}\right) .
$$

The possibility to solve the above equation depends on the form of $\Gamma^{\Upsilon}{ }_{\Lambda \Delta}$. For the metric given by the relation (3.8) we find the general explicit solution with the initial condition that will produce the Riemannian coordinates. The initial condition for producing the flat supercoordinates, in which the metric requires the constant form $\bar{G}(\Omega)=G(\Phi=0)$, is given by

$$
\left.\frac{\Omega^{A} \overleftarrow{\partial}}{\partial \Phi^{\Upsilon}}\right|_{\Phi=0}=\delta_{\Upsilon}^{A}
$$

To continue, we shall present the solution of equations (4.2) in detail for the metric (3.8).

\section{Solving the equations for flat supercoordinates}

In this section we find the transformation of supercoordinates that makes the metric constant and use it for solving the models. As mentioned in subsection (3.1), the model given by the metric (3.8) lives in the flat background in the sense that its Riemann tensor vanishes. In spite of the fact that the metric is flat, the $\Gamma^{\Upsilon}{ }_{\Lambda \Delta}$ are not zero, so, it is not easy to find the coordinates $\Phi^{\Upsilon}(\tau, \sigma)$ that solve the equations of motion given by the action (2.1). After the use of the relation (2.2) to calculate the components of the connection for the metric (3.8), equations (4.2) then read

$$
\begin{aligned}
-\frac{\partial \Omega^{A}}{\partial y} & =\frac{\partial^{2} \Omega^{A}}{\partial x \partial y}, \\
-e^{x} \frac{\partial \Omega^{A}}{\partial y} & =\frac{\partial^{2} \Omega^{A}}{\partial \psi \partial \chi}, \\
\frac{\partial \Omega^{A}}{\partial x}+2 y \frac{\partial \Omega^{A}}{\partial y} & =\frac{\partial^{2} \Omega^{A}}{\partial x^{2}}, \\
\frac{\partial \Omega^{A}}{\partial y} e^{x} \chi-(-1)^{A} \frac{\partial \Omega^{A}}{\partial \psi} & =-(-1)^{A} \frac{\partial^{2} \Omega^{A}}{\partial x \partial \psi}, \\
\frac{\partial^{2} \Omega^{A}}{\partial y^{2}}=0, \quad \frac{\partial^{2} \Omega^{A}}{\partial \psi^{2}}=0, \frac{\partial^{2} \Omega^{A}}{\partial \chi^{2}} & =0, \\
\frac{\partial^{2} \Omega^{A}}{\partial x \partial \chi}=0, \quad \frac{\partial^{2} \Omega^{A}}{\partial y \partial \psi}=0, \quad \frac{\partial^{2} \Omega^{A}}{\partial y \partial \chi} & =0,
\end{aligned}
$$


where $(-1)^{A}=\left\{\begin{array}{ll}1 & A=1,2 \\ -1 & A=3,4\end{array}\right.$. The above equations can be solved and the general solution is

$$
\begin{aligned}
& \Omega^{1}=a_{0}+a_{1}\left(y e^{-x}+\psi \chi\right)+a_{2} e^{x}, \\
& \Omega^{2}=b_{0}+b_{1}\left(y e^{-x}+\psi \chi\right)+b_{2} e^{x}, \\
& \Omega^{3}=\alpha_{0}+c_{1} \chi+c_{2} e^{x} \psi, \\
& \Omega^{4}=\beta_{0}+d_{1} \chi+d_{2} e^{x} \psi,
\end{aligned}
$$

where the integration constants $\alpha_{0}$ and $\beta_{0}$ are real $a$-numbers (odd real constants) while the rest of constants are real $c$-numbers (even real constants) [29]. Imposing the initial condition (4.3), the integration constants will be determined. Then, the solution (5.7) takes the following form

$$
\begin{aligned}
& \Omega^{1}=a_{0}+e^{x}, \\
& \Omega^{2}=b_{0}+y e^{-x}+\psi \chi, \\
& \Omega^{3}=\alpha_{0}+e^{x} \psi, \\
& \Omega^{4}=\beta_{0}+\chi .
\end{aligned}
$$

In the following, for simplicity we will assume that $\alpha_{0}=\beta_{0}=0$. The transformation (5.8) transforms the metric (3.8) into constant one

$$
\bar{G}_{A B}(\Omega)=\left(\begin{array}{cccc}
0 & K & 0 & 0 \\
K & 0 & 0 & 0 \\
0 & 0 & 0 & K \\
0 & 0 & -K & 0
\end{array}\right) .
$$

Thus, the action corresponding to the metric (5.9) is given by

$$
\begin{aligned}
\bar{S} & =\frac{1}{2} \int d \sigma^{+} d \sigma^{-}(-1)^{A} \partial_{+} \Omega^{A} \bar{G}_{A B} \partial_{-} \Omega^{B} \\
& =\frac{K}{2} \int d \sigma^{+} d \sigma^{-}\left(\partial_{+} \Omega^{1} \partial_{-} \Omega^{2}+\partial_{+} \Omega^{2} \partial_{-} \Omega^{1}-\partial_{+} \Omega^{3} \partial_{-} \Omega^{4}+\partial_{+} \Omega^{4} \partial_{-} \Omega^{3}\right) .
\end{aligned}
$$

Now, we show that the transformation (5.8) is indeed a classical supercanonical transformation.

\subsection{Supercoordinates transformation as a supercanonical transformation}

This subsection begins with the calculation of the momentums corresponding to the actions (3.7) and (5.10) and the transformation between them. To this end, we first write Lagrangians of the actions (3.7) and (5.10) in the worldsheet coordinates. Using the definition of the conjugate momentum of $\Phi^{\Upsilon}$ on the supermanifolds as

$$
P_{\Phi^{\Upsilon}}=\frac{L \overleftarrow{\delta}}{\delta\left(\partial_{\tau} \Phi^{\Upsilon}\right)}
$$


the corresponding moments are then found to be

$$
\begin{array}{llrl}
P_{x} & =K\left(-2 y \partial_{\tau} x+\partial_{\tau} y-e^{x} \chi \partial_{\tau} \psi\right), & & P_{y}=K \partial_{\tau} x, \\
P_{\psi} & =K\left(-e^{x} \partial_{\tau} x \chi+e^{x} \partial_{\tau} \chi\right), & & P_{\chi}=-K e^{x} \partial_{\tau} \psi,
\end{array}
$$

for the model (3.7) and

$$
\begin{array}{ll}
\bar{P}_{\Omega^{1}}=K \partial_{\tau} \Omega^{2}, & \bar{P}_{\Omega^{2}}=K \partial_{\tau} \Omega^{1}, \\
\bar{P}_{\Omega^{3}}=K \partial_{\tau} \Omega^{4}, & \bar{P}_{\Omega^{4}}=-K \partial_{\tau} \Omega^{3},
\end{array}
$$

for the model (5.10). The transformation (5.8) is a transformation between supercoordinates $\Phi^{\Upsilon}$ and $\Omega^{A}$. Utilizing the transformation (5.8) and contracting relations (5.12) and (5.13), the transformation between momentums is also obtained to be of the form

$$
\begin{array}{llrl}
\bar{P}_{\Omega^{1}} & =e^{-x}\left(P_{x}+y P_{y}+\psi P_{\psi}\right)+\psi \chi P_{y}, & & \bar{P}_{\Omega^{2}}=e^{x} P_{y}, \\
\bar{P}_{\Omega^{3}}=e^{-x} P_{\psi}+\chi P_{y}, & & \bar{P}_{\Omega^{4}}=-e^{x} \psi P_{y}+P_{\chi} .
\end{array}
$$

It can be useful to comment on the fact that the graded Poisson brackets are preserved when equations (5.14) are combined with (5.8). The basic equal-time graded Poisson brackets for the pair of supercanonical variables $\left(\Phi^{\Upsilon}, P_{\Lambda}\right)$ are given by

$$
\begin{aligned}
& \left\{\Phi^{\Upsilon}, P_{\Lambda}\right\}=\delta^{\Upsilon}{ }_{\Lambda} \delta\left(\sigma-\sigma^{\prime}\right), \\
& \left\{\Phi^{\Upsilon}, \Phi^{\Lambda}\right\}=\left\{P_{\Upsilon}, P_{\Lambda}\right\}=0 .
\end{aligned}
$$

It is understood that the first one on the bracket is always evaluated at $\sigma$ and the second one at $\sigma^{\prime}$. Using equations (5.14) and (5.8) together with (5.15) one can show that the equal-time graded Poisson brackets for the pair of supercanonical variables $\left(\Omega^{A}, \bar{P}_{B}\right)$ are also preserved; that is,

$$
\begin{aligned}
& \left\{\Omega^{A}, \bar{P}_{B}\right\}=\delta^{A}{ }_{B} \delta\left(\sigma-\sigma^{\prime}\right), \\
& \left\{\Omega^{A}, \Omega^{B}\right\}=\left\{\bar{P}_{A}, \bar{P}_{B}\right\}=0 .
\end{aligned}
$$

Let us now to obtain the Hamiltonian corresponding to the action (5.10). Utilizing relation (5.13) we get

$$
\overline{\mathcal{H}}(\Omega)=K\left(\partial_{\tau} \Omega^{1} \partial_{\tau} \Omega^{2}+\partial_{\sigma} \Omega^{1} \partial_{\sigma} \Omega^{2}-\partial_{\tau} \Omega^{3} \partial_{\tau} \Omega^{4}-\partial_{\sigma} \Omega^{3} \partial_{\sigma} \Omega^{4}\right) .
$$

Under the transformation (5.8), the Hamiltonian (5.17) turns into

$$
\begin{aligned}
\mathcal{H}(\Phi)= & K\left(\partial_{\tau} x \partial_{\tau} y+\partial_{\sigma} x \partial_{\sigma} y-y \partial_{\tau} x \partial_{\tau} x-y \partial_{\sigma} x \partial_{\sigma} x\right. \\
& \left.-e^{x} \partial_{\tau} x \chi \partial_{\tau} \psi-e^{x} \partial_{\sigma} x \chi \partial_{\sigma} \psi-e^{x} \partial_{\tau} \psi \partial_{\tau} \chi-e^{x} \partial_{\sigma} \psi \partial_{\sigma} \chi\right) .
\end{aligned}
$$

One can simply show that the Hamiltonian (5.18) is nothing but the Hamiltonian corresponding to the action (3.7). Thus, we proved that the Hamiltonians corresponding 
to the actions (3.7) and (5.10) are equal; that is, under the transformation of supercoordinates (5.8), one goes from $\overline{\mathcal{H}}(\Omega)$ to $\mathcal{H}(\Phi)$ and vice versa, hence, proving that the transformation (5.8) or (5.14) is indeed a supercanonical transformation.

A bit surprisingly, one can show that under the linear transformation

$$
\begin{aligned}
{\Omega^{\prime}}^{1} & =\frac{1}{\sqrt{2}}\left(K \Omega^{2}-\Omega^{1}\right), \\
{\Omega^{\prime}}^{2} & =\frac{1}{\sqrt{2}}\left(K \Omega^{2}+\Omega^{1}\right), \\
\Omega^{\prime 3} & =-\Omega^{3} \\
{\Omega^{\prime}}^{4} & =-K \Omega^{4}
\end{aligned}
$$

the action (5.10) reduces to the following action

$$
S^{\prime}=\frac{1}{2} \int d \sigma^{+} d \sigma^{-}\left(\partial_{+} \Omega^{\prime \mu} \partial_{-} \Omega^{\prime \nu} \eta_{\mu \nu}+\partial_{+} \Omega^{\prime \alpha} \partial_{-} \Omega^{\prime \beta} G_{\beta \alpha}\right)
$$

where the indices $\mu, \nu$ and $\alpha, \beta$ range over the values 1,2 and 3,4 , respectively. So, $\eta_{\mu \nu}=$ $\operatorname{diag}(-1,1)$ denotes the Minkowski metric of the bosonic directions, and the metric of the fermionic directions is

$$
G_{\beta \alpha}=\left(\begin{array}{cc}
0 & 1 \\
-1 & 0
\end{array}\right) .
$$

The action (5.20) is nothing but the Polyakov action on a (2|2)-dimensional flat supermanifold [34]. Meanwhile, one can show that the linear transformation (5.19) is also a supercanonical transformation.

\section{Calculation of the dual solution using the super Poisson-Lie T-duality transformations}

The relation between the solutions $\Phi^{\Upsilon}\left(\sigma^{+}, \sigma^{-}\right)$of the original model $(3.7)$ and $\tilde{\Phi}^{\Upsilon}\left(\sigma^{+}, \sigma^{-}\right)$ of the dual model (3.14) is given by two possible decompositions (2.10) and (2.25), in which the $\tilde{h}$ satisfies the equations (2.19) and (2.20). Therefore, for a solution $\Phi^{\Upsilon}\left(\sigma^{+}, \sigma^{-}\right)$of the sigma model we must find $\tilde{h}\left(\sigma^{+}, \sigma^{-}\right)$, i.e., solve the system of PDEs (2.19) and (2.20). Then, using the transformations (2.10) and (2.25) we find the solutions $\tilde{\Phi}^{\Upsilon}\left(\sigma^{+}, \sigma^{-}\right)$of the dual sigma model. To this end, by introducing

$$
\begin{aligned}
\Omega^{a} & =W^{a}\left(\sigma^{+}\right)+U^{a}\left(\sigma^{-}\right), & & a=1,2, \\
\Omega^{\alpha} & =\eta^{\alpha}\left(\sigma^{+}\right)+\xi^{\alpha}\left(\sigma^{-}\right), & & \alpha=3,4,
\end{aligned}
$$

we transform the equations of motion to the wave equations. In relations (6.1) and (6.2), the $W^{a}\left(\sigma^{+}\right)$and the $U^{a}\left(\sigma^{-}\right)$are even, arbitrary functions while the $\eta^{\alpha}\left(\sigma^{+}\right)$and the $\xi^{\alpha}\left(\sigma^{-}\right)$ 
are odd, arbitrary ones. Thus, functions $\Phi^{\Upsilon}\left(\sigma^{+}, \sigma^{-}\right)$follow from (5.8), (6.1) and (6.2)

$$
\begin{aligned}
e^{x}= & W^{1}\left(\sigma^{+}\right)+U^{1}\left(\sigma^{-}\right)-a_{0}, \\
y= & \left(W^{1}\left(\sigma^{+}\right)+U^{1}\left(\sigma^{-}\right)-a_{0}\right)\left(W^{2}\left(\sigma^{+}\right)+U^{2}\left(\sigma^{-}\right)-b_{0}\right) \\
& -\left(\eta^{3}\left(\sigma^{+}\right)+\xi^{3}\left(\sigma^{-}\right)\right)\left(\eta^{4}\left(\sigma^{+}\right)+\xi^{4}\left(\sigma^{-}\right)\right), \\
\psi= & \frac{\eta^{3}\left(\sigma^{+}\right)+\xi^{3}\left(\sigma^{-}\right)}{W^{1}\left(\sigma^{+}\right)+U^{1}\left(\sigma^{-}\right)-a_{0}}, \\
\chi= & \eta^{4}\left(\sigma^{+}\right)+\xi^{4}\left(\sigma^{-}\right) .
\end{aligned}
$$

Now one can show that the above functions satisfy the following equations

$$
\begin{aligned}
0= & \partial_{+} \partial_{-} x+\partial_{-} x \partial_{+} x, \\
0= & \partial_{+} \partial_{-} y+2 y \partial_{-} x \partial_{+} x-\partial_{-} y \partial_{+} x-\partial_{-} x \partial_{+} y \\
& +e^{x} \chi\left(\partial_{-} \psi \partial_{+} x+\partial_{-} x \partial_{+} \psi\right)+e^{x}\left(\partial_{-} \psi \partial_{+} \chi-\partial_{-} \chi \partial_{+} \psi\right), \\
0= & \partial_{+} \partial_{-} \psi+\partial_{-} x \partial_{+} \psi+\partial_{-} \psi \partial_{+} x, \\
0= & \partial_{+} \partial_{-} \chi .
\end{aligned}
$$

Note that equations (6.4) are the equations of motion of action (3.7) which have been obtained by using relation (2.4). Thus, we could find the solution of the equations of motion of the original model by the use of the flat supercoordinates transformation. In the following to find $\tilde{h}\left(\sigma^{+}, \sigma^{-}\right)$, we solve the system of PDEs (2.19) and (2.20). To make up the right hand sides of the equations (2.19) and (2.20) one must use (3.5) to calculate ${ }_{i} V^{\Upsilon}$ 's. Then, using (3.8) the right hand sides read

$$
\begin{aligned}
& \left(\partial_{ \pm} \tilde{h} \tilde{h}^{-1}\right)_{1}= \pm K\left[-2\left(y \partial_{ \pm} x+\psi \chi \partial_{ \pm} e^{x}\right)+\partial_{ \pm} y+\partial_{ \pm}\left(e^{x} \psi \chi\right)\right] \\
& \left(\partial_{ \pm} \tilde{h} \tilde{h}^{-1}\right)_{2}= \pm K \partial_{ \pm} e^{x} \\
& \left(\partial_{ \pm} \tilde{h} \tilde{h}^{-1}\right)_{3}= \pm K\left(\chi \partial_{ \pm} e^{x}-e^{x} \partial_{ \pm} \chi\right) \\
& \left(\partial_{ \pm} \tilde{h} \tilde{h}^{-1}\right)_{4}= \pm K \partial_{ \pm}\left(e^{x} \psi\right) .
\end{aligned}
$$

On the other hand, if we parametrize elements of the Abelian Lie supergroup $\tilde{G}$ with bosonic coordinates $\left\{\tilde{h}_{1}, \tilde{h}_{2}\right\}$ and fermionic ones $\left\{\tilde{h}_{3}, \tilde{h}_{4}\right\}$ as

$$
\tilde{h}=e^{\tilde{h}_{4} \tilde{X}^{4}} e^{\tilde{h}_{2} \tilde{X}^{2}} e^{\tilde{h}_{1} \tilde{X}^{1}} e^{\tilde{h}_{3} \tilde{X}^{3}},
$$

then, the left hand sides are just $\partial_{ \pm} \tilde{h}_{i}$. Substituting the solution $\Phi^{\Upsilon}\left(\sigma^{+}, \sigma^{-}\right)$given by $(6.3)$ into (6.5), the system of PDEs (2.19) and (2.20) can be solved and the general solution is ${ }^{7}$

$$
\begin{aligned}
& \tilde{h}_{1}=K\left[Q_{ \pm}+U_{-}^{1} W_{+}^{2}-U_{-}^{2} W_{+}^{1}+b_{0}\left(W_{+}^{1}-U_{-}^{1}\right)-a_{0}\left(W_{+}^{2}-U_{-}^{2}\right)\right]+c_{0}, \\
& \tilde{h}_{2}=K\left(W_{+}^{1}-U_{-}^{1}\right)+d_{0}, \\
& \tilde{h}_{3}=K\left[\gamma_{ \pm}+W_{+}^{1} \xi_{-}^{4}-U_{-}^{1} \eta_{+}^{4}+a_{0}\left(\eta_{+}^{4}-\xi_{-}^{4}\right)\right]+\zeta_{0}, \\
& \tilde{h}_{4}=K\left(\eta_{+}^{3}-\xi_{-}^{3}\right)+\varrho_{0},
\end{aligned}
$$

\footnotetext{
${ }^{7}$ Here, $Q_{ \pm}$and $W_{+}^{a}$ have been used instead of $Q\left(\sigma^{+}, \sigma^{-}\right)$and $W^{a}\left(\sigma^{+}\right)$, respectively. The other notations follow this rule, too.
} 
where $c_{0}$ and $d_{0}$ are even real constants while $\zeta_{0}$ and $\varrho_{0}$ are odd real ones, and the functions $Q_{ \pm}$and $\gamma_{ \pm}$solve

$$
\begin{aligned}
\partial_{+} Q_{ \pm} & =W_{+}^{1} W_{+}^{2 \prime}-W_{+}^{1^{\prime}} W_{+}^{2} \\
\partial_{-} Q_{ \pm} & =U_{-}^{1^{\prime}} U_{-}^{2}-U_{-}^{1}{U_{-}^{2}}^{\prime} \\
\partial_{+} \gamma_{ \pm} & =W_{+}^{1 \prime} \eta_{+}^{4}-W_{+}^{1} \eta_{+}^{{ }^{\prime}} \\
\partial_{-} \gamma_{ \pm} & =U_{-}^{1} \xi_{-}^{4}-U_{-}^{1} \xi_{-}^{4}
\end{aligned}
$$

in which primes denote differentiation with respect to the arguments.

At the end of this section, we get the solution of the equations of motion for the dual sigma model in the curved background (3.15) by using the duality transformation that follows two possible decompositions of elements of $l \in D$ as

$$
l\left(\sigma^{+}, \sigma^{-}\right)=g\left(\sigma^{+}, \sigma^{-}\right) \tilde{h}\left(\sigma^{+}, \sigma^{-}\right)=\tilde{g}\left(\sigma^{+}, \sigma^{-}\right) h^{\prime}\left(\sigma^{+}, \sigma^{-}\right), \quad h^{\prime}\left(\sigma^{+}, \sigma^{-}\right) \in G .
$$

If we write all elements of the Lie supergroups $G$ and $\tilde{G}$ as a product of elements of oneparametric sub-(super)groups, then, the equation (6.9) yields

$$
e^{\chi X_{4}} e^{y X_{2}} e^{x X_{1}} e^{\psi X_{3}} e^{\tilde{h}_{4} \tilde{X}^{4}} e^{\tilde{h}_{2} \tilde{X}^{2}} e^{\tilde{h}_{1} \tilde{X}^{1}} e^{\tilde{h}_{3} \tilde{X}^{3}}=e^{\tilde{\chi} \tilde{X}^{4}} e^{\tilde{y} \tilde{X}^{2}} e^{\tilde{x} \tilde{X}^{1}} e^{\tilde{\psi} \tilde{X}^{3}} e^{h^{\prime 4} X_{4}} e^{h^{\prime 2} X_{2}} e^{h^{\prime 1} X_{1}} e^{h^{\prime 3} X_{3}} .
$$

Now, using the (anti)commutation relations of the Drinfel'd superdouble $\left(\left(\mathcal{C}_{1}^{1}+\mathcal{A}\right), \mathcal{I}_{(2 \mid 2)}\right)$ obtained in section 3 , the right hand side of the equation (6.10) is rewritten in the form

$$
\begin{aligned}
& e^{h^{\prime 4} X_{4}} e^{h^{\prime 2} X_{2}} e^{h^{\prime 1} X_{1}} e^{h^{\prime 3} X_{3}} e^{\left(\tilde{\chi}+\tilde{y} e^{h^{1}} h^{\prime 3}\right) \tilde{X}} e^{4} e^{h^{h^{\prime 1}}} \tilde{X}^{2} \\
& \times e^{\left(\tilde{x}-\tilde{y} h^{\prime 2}+\tilde{y} e^{h^{1}} h^{\prime 4} h^{\prime 3}+e^{h^{\prime \prime}} \tilde{\psi} h^{\prime 3}\right) \tilde{X}{ }^{1}} e^{\left(\tilde{y} h^{\prime 4}+\tilde{\psi}\right) e^{h^{\prime 1}} \tilde{X}^{3}} .
\end{aligned}
$$

Hence, we get

$$
\begin{aligned}
\tilde{x} & =\tilde{h}_{1}-\tilde{h}_{3} \psi+y e^{-x} \tilde{h}_{2}, \\
\tilde{y} & =e^{-x} \tilde{h}_{2}, \\
\tilde{\psi} & =e^{-x}\left(\tilde{h}_{3}-\tilde{h}_{2} \chi\right), \\
\tilde{\chi} & =\tilde{h}_{4}-\tilde{h}_{2} \psi,
\end{aligned}
$$

and

$$
h^{\prime 1}=x, \quad h^{\prime 2}=y, \quad h^{3}=\psi, \quad h^{4}=\chi
$$

Inserting relations (6.3) and (6.7) into (6.12) we get the solution of the equations of motion 
for the dual sigma model given by the action (3.14) as follows:

$$
\begin{aligned}
\tilde{x} & =K\left[Q_{ \pm}+W_{+}^{1} W_{+}^{2}-U_{-}^{1} U_{-}^{2}-\frac{\left(\gamma_{ \pm}-W_{+}^{1} \eta_{+}^{4}+U_{-}^{1} \xi_{-}^{4}\right)\left(\eta_{+}^{3}+\xi_{-}^{3}\right)}{W_{+}^{1}+U_{-}^{1}}\right], \\
\tilde{y} & =K\left(\frac{W_{+}^{1}-U_{-}^{1}}{W_{+}^{1}+U_{-}^{1}}\right), \\
\tilde{\psi} & =\frac{K}{W_{+}^{1}+U_{-}^{1}}\left(\gamma_{ \pm}+U_{-}^{1} \xi_{-}^{4}-W_{+}^{1} \eta_{+}^{4}\right), \\
\tilde{\chi} & =\frac{2 K}{W_{+}^{1}+U_{-}^{1}}\left(U_{-}^{1} \eta_{+}^{3}-W_{+}^{1} \xi_{-}^{3}\right),
\end{aligned}
$$

where we have assumed that the constants $a_{0}, b_{0}, c_{0}, d_{0}$ and $\zeta_{0}, \varrho_{0}$ are zero.

Applying the formula (2.4) for the dual model, the equations of motion of the sigma model given by the curved background (3.15) are obtained to be of the form

$$
\begin{array}{r}
\partial_{+} \partial_{-} \tilde{x}+\left(\frac{1}{K+\tilde{y}}\right) \partial_{-} \tilde{\chi} \partial_{+} \tilde{\psi}+\left(\frac{1}{K-\tilde{y}}\right) \partial_{-} \tilde{\psi} \partial_{+} \tilde{\chi}=0, \\
\partial_{+} \partial_{-} \tilde{y}+2\left(\frac{\tilde{y}}{K^{2}-\tilde{y}^{2}}\right) \partial_{+} \tilde{y} \partial_{-} \tilde{y}=0, \\
\partial_{+} \partial_{-} \tilde{\psi}+\left(\frac{1}{K-\tilde{y}}\right) \partial_{-} \tilde{\psi} \partial_{+} \tilde{y}-\left(\frac{1}{K+\tilde{y}}\right) \partial_{-} \tilde{y} \partial_{+} \tilde{\psi}=0, \\
\partial_{+} \partial_{-} \tilde{\chi}-\left(\frac{1}{K+\tilde{y}}\right) \partial_{-} \tilde{\chi} \partial_{+} \tilde{y}+\left(\frac{1}{K-\tilde{y}}\right) \partial_{-} \tilde{y} \partial_{+} \tilde{\chi}=0 .
\end{array}
$$

Due to the complexity of the above equations, we are unable to solve them directly. But we have found their solution in the form (6.14) by using the flat supercoordinates transformation. One can check that solution (6.14) actually satisfies the equations of motion (6.15).

\section{Invariance of the dilaton field under the supercoordinates transforma- tion and conformal sigma models}

To guarantee the conformal invariance of the sigma models, at least at the one-loop level, one must show that they satisfy the vanishing $\beta$-function equations. To this end, we need to add a scalar field $\varphi$ (dilaton field) to the action (2.1) and rewrite it in the usual standard form $[25]$

$$
S=\frac{1}{2} \int d \sigma^{+} d \sigma^{-}\left[(-1)^{\Upsilon} \partial_{+} \Phi^{\Upsilon} \mathcal{E}_{\Upsilon \Lambda}(\Phi) \partial_{-} \Phi^{\Lambda}-\frac{1}{4} R^{(2)} \varphi\right],
$$

where $R^{(2)}$ is the curvature of the worldsheet. It is well known that the connection between the string effective action and the sigma model (7.1) is through the Weyl anomaly coefficients [25]

$$
\begin{aligned}
& \beta_{\Upsilon \Lambda}^{(G)}:=R_{\Upsilon \Lambda}+\frac{1}{4} H_{\Upsilon \Delta \Xi} H^{\Xi \Delta}+2 \vec{\nabla}_{\Upsilon} \vec{\nabla}_{\Lambda} \varphi=0, \\
& \beta_{\Upsilon \Lambda}^{(B)}:=(-1)^{\Delta} \vec{\nabla}^{\Delta}\left(e^{-2 \varphi} H_{\Delta \Upsilon \Lambda}\right)=0, \\
& \beta^{(\varphi)}:=-R-\frac{1}{12} H_{\Upsilon \Lambda \Delta} H^{\Delta \Lambda \Upsilon}+4 \vec{\nabla}_{\Upsilon} \varphi \vec{\nabla}^{\Upsilon} \varphi-4 \vec{\nabla}_{\Upsilon} \vec{\nabla}^{\Upsilon} \varphi=0,
\end{aligned}
$$


where $R_{\Upsilon \Lambda}$ and $R$ are the Ricci tensor and the scalar curvature, respectively, and $H_{\Upsilon_{\Lambda} \Delta}$ is defined by equation (2.3). As shown in [25], the vanishing of $\beta$-function equations can be derived as the equations of motion of the string effective action on the supermanifold $M$

$$
S_{\text {eff }}=\int d^{(m, n)} \Phi \sqrt{G} e^{-2 \varphi}\left(R+4 \vec{\nabla}_{\Upsilon} \varphi \vec{\nabla}^{\Upsilon} \varphi+\frac{1}{12} H_{\Upsilon \Lambda \Delta} H^{\Delta \Lambda \Upsilon}\right),
$$

where $(m, n)=\left(d_{B} \mid d_{F}\right)$ and $G=\operatorname{sdet}\left({ }_{\Upsilon} G_{\Lambda}\right)$. As shown in section 5, the action (5.10) has been expressed in terms of the flat supercoordinates $\Omega^{A}$ transforming the flat metric $G_{\Upsilon \Lambda}(\Phi)$ into a constant form $G_{A B}(\Omega)$. Moreover, the model is torsionless. Therefore, $\bar{\Gamma}_{B C}^{A}$ and $\bar{H}_{A B C}$ are vanished. Consequently, equation (7.3) is satisfied and equations (7.2) and (7.4) are, respectively, read

$$
\begin{aligned}
& 0=\left(\frac{\bar{\varphi} \overleftarrow{\partial}}{\partial \Omega^{A}}\right) \frac{\overleftarrow{\partial}}{\partial \Omega^{B}} \\
& 0=(-1)^{B} \frac{\bar{\varphi} \overleftarrow{\partial}}{\partial \Omega^{A}} \bar{G}^{A B} \frac{\bar{\varphi} \overleftarrow{\partial}}{\partial \Omega^{B}}-\left(\frac{\bar{\varphi} \overleftarrow{\partial}}{\partial \Omega^{A}}\right) \frac{\overleftarrow{\partial}}{\partial \Omega^{B}} \bar{G}^{B A}
\end{aligned}
$$

It is clear that for the metric constant (5.9) and a constant dilaton field, the vanishing $\beta$-function equations (equations (7.6) and (7.7)) are satisfied. But, since we know the flat supercoordinates of the model, we can easily find the general form of the dilaton field that together with the metric constant (5.9) satisfy the equations (7.2)-(7.4). From the form of equations (7.6) and (7.7) it is easy to see that the general form of their dilaton field is

$$
\bar{\varphi}(\Omega)=\bar{\varphi}_{0}+\frac{\lambda_{1} \lambda_{2}}{k_{0}} \Omega^{1}+k_{0} \Omega^{2}+\lambda_{1} \Omega^{3}+\lambda_{2} \Omega^{4},
$$

where $\bar{\varphi}_{0}$ and $k_{0}$ are even real constants while $\lambda_{1}$ and $\lambda_{2}$ are odd real ones. Now, inserting the transformation (5.8) into (7.8), one gets the general form of the dilaton field of the original sigma model (the model described by the metric (3.8)) in such a way that the result is

$$
\varphi(\Phi)=\varphi_{0}+\frac{\lambda_{1} \lambda_{2}}{k_{0}} e^{x}+k_{0}\left(y e^{-x}+\psi \chi\right)+\lambda_{1} \psi e^{x}+\lambda_{2} \chi
$$

where $\varphi_{0}=\bar{\varphi}_{0}+\frac{\lambda_{1} \lambda_{2}}{k_{0}} a_{0}+k_{0} b_{0}+\lambda_{1} \alpha_{0}+\lambda_{2} \beta_{0}$.

On the other hand, as explained in subsection (3.1), the original model is flat (its the Ricci tensor and the scalar curvature are zero) and also torsionless. So, for this model, equation (7.3) is satisfied and equations (7.2) and (7.4) are, respectively, turned into

$$
\begin{aligned}
& 0=\left(\frac{\varphi \overleftarrow{\partial}}{\partial \Phi^{\Upsilon}}\right) \frac{\overleftarrow{\partial}}{\partial \Phi^{\Lambda}}-\left(\frac{\varphi \overleftarrow{\partial}}{\partial \Phi^{\Delta}}\right) \Gamma_{\Upsilon \Lambda}^{\Delta} \\
& 0=(-1)^{\Lambda} \frac{\varphi \overleftarrow{\partial}}{\partial \Phi^{\Upsilon}} G^{\Upsilon \Lambda} \frac{\varphi \overleftarrow{\partial}}{\partial \Phi^{\Lambda}}-\left[\left(\frac{\varphi \overleftarrow{\partial}}{\partial \Phi^{\Upsilon}}\right) \frac{\overleftarrow{\partial}}{\partial \Phi^{\Lambda}}-\left(\frac{\varphi \overleftarrow{\partial}}{\partial \Phi^{\Delta}}\right) \Gamma_{\Upsilon \Lambda}^{\Delta}\right] G^{\Lambda \Upsilon}
\end{aligned}
$$

It is interesting to see that the dilaton field (7.9) is the general solution of equations (7.10) and (7.11); that is, the metric (3.8) together with the dilaton field (7.9) satisfy the vanishing 
$\beta$-function equations. Thus, we conclude that the models (3.7) and (5.10) are conformally invariant up to one-loop. We also showed that under the transformation of supercoordinates (5.8), one goes from $\varphi(\Phi)$ to $\bar{\varphi}(\Omega)$ and vice versa, i.e., the dilaton field is invariant under the transformation (5.8).

Let us now turn into the dual model. For the dual model with the background (3.15), we find that the only non-zero component of $\tilde{R}_{\Upsilon \Lambda}$ is $\tilde{R}_{22}=\frac{2 K^{2}}{\left(K^{2}-\tilde{y}^{2}\right)^{2}}$; as $\tilde{G}^{22}=0, \tilde{R}=0$. Also, one quickly finds that the only non-zero component of $\tilde{H}$ is $\tilde{H}_{234}=-\frac{K^{2}}{\left(K^{2}-\tilde{y}^{2}\right)^{2}}$. It is then straightforward to verify that $\tilde{H}_{\Upsilon \Lambda \Delta} \tilde{H}^{\Delta \Lambda \Upsilon}=0$, and the only non-zero component of $\tilde{H}_{\Upsilon \Delta \Xi} \tilde{H}_{\Lambda}^{\Xi \Delta}$ is $\tilde{H}_{234} \tilde{H}_{2}^{43}=-\frac{K^{2}}{\left(K^{2}-\tilde{y}^{2}\right)^{2}}$. Putting these in equations $(7.2)-(7.4)$ we arrive at ${ }^{8}$

$$
\begin{aligned}
& 0=\tilde{R}_{\Upsilon \Lambda}+\frac{1}{2} \tilde{H}_{\Upsilon 34} \tilde{H}^{43}{ }_{\Lambda}+2\left[\left(\frac{\tilde{\varphi} \overleftarrow{\partial}}{\partial \tilde{\Phi}^{\Upsilon}}\right) \frac{\overleftarrow{\partial}}{\partial \tilde{\Phi}^{\Lambda}}-\left(\frac{\tilde{\varphi} \overleftarrow{\partial}}{\partial \tilde{\Phi}^{\Delta}}\right) \tilde{\Gamma}_{\Upsilon \Lambda}^{\Delta}\right] \\
& 0=-2(-1)^{\Delta}\left(\frac{\tilde{\varphi} \overleftarrow{\partial}}{\partial \tilde{\Phi}^{\Xi}}\right) \tilde{G}^{\Xi \Delta} \tilde{H}_{\Delta \Upsilon \Lambda}+(-1)^{\Delta+\Xi+\Xi(\Upsilon+\Lambda)} \tilde{G}^{\Xi \Delta}\left(\tilde{H}_{\Delta \Upsilon \Lambda} \overleftarrow{\nabla}_{\Xi}\right) \\
& 0=(-1)^{\Lambda} \frac{\tilde{\varphi} \overleftarrow{\partial}}{\partial \tilde{\Phi}^{\Upsilon}} \tilde{G}^{\Upsilon \Lambda} \frac{\tilde{\varphi} \overleftarrow{\partial}}{\partial \tilde{\Phi}^{\Lambda}}-\left[\left(\frac{\tilde{\varphi} \overleftarrow{\partial}}{\partial \tilde{\Phi}^{\Upsilon}}\right) \frac{\overleftarrow{\partial}}{\partial \tilde{\Phi}^{\Lambda}}-\left(\frac{\tilde{\varphi} \overleftarrow{\partial}}{\partial \tilde{\Phi}^{\Delta}}\right) \tilde{\Gamma}_{\Upsilon \Lambda}^{\Delta}\right] \tilde{G}^{\Lambda \Upsilon}
\end{aligned}
$$

By solving the above equations, one gets the general form of the dilaton field of the dual model to be of the form

$$
\tilde{\Phi}=\tilde{\Phi}_{0}+\frac{\tilde{k}_{0}}{2 K} \ln \left|\frac{K+\tilde{y}}{K-\tilde{y}}\right|-\frac{3}{32}\left(\ln \left|\frac{K+\tilde{y}}{K-\tilde{y}}\right|\right)^{2},
$$

where $\tilde{\Phi}_{0}$ and $\tilde{k}_{0}$ are the even constants of integration. Thus, conformal invariance of the dual model is also guaranteed up to one-loop.

\section{Conclusion}

In the present work, we have obtained the solution of the equations of motion for a super non-Abelian T-dual sigma model in curved background by the super Poisson-Lie T-duality. In this way, we have first used the fact that the super Poisson-Lie T-duality transformations can be explicitly performed for the Drinfel'd superdouble $\left(\left(C_{1}^{1}+A\right), I_{(2 \mid 2)}\right)$. Then, we have obtained the explicit transformation between the supergroup coordinates of the model living in the flat background and its flat supercoordinates. Also, we have proved that flat supercoordinates transformation is a supercanonical transformation. Furthermore, we have found a linear transformation of the flat supercoordinates which reduces the action of the flat model with the metric constant to the Polyakov action on a (2|2)-dimensional flat supermanifold. By reducing the transformation of supercoordinates to the solution

\footnotetext{
${ }^{8}$ Note that the right covariant derivative of the components of a three-form on a supermanifold $M$ is given by

$$
\tilde{H}_{\Delta \Upsilon \Lambda} \overleftarrow{\nabla}_{\Xi}=\tilde{H}_{\Delta \Upsilon \Lambda} \frac{\overleftarrow{\partial}}{\partial \tilde{\Phi}^{\Xi}}-(-1)^{(\Upsilon+\Lambda)(\Delta+\Omega)} \tilde{H}_{\Omega \Upsilon \Lambda} \tilde{\Gamma}_{\Delta \Xi}^{\Omega}-(-1)^{\Lambda(\Upsilon+\Omega)} \tilde{H}_{\Delta \Omega \Lambda} \tilde{\Gamma}_{\Upsilon \Xi}^{\Omega}-\tilde{H}_{\Delta \Upsilon \Omega} \tilde{\Gamma}_{\Lambda \Xi}^{\Omega}
$$


of the wave equation and using the super Poisson-Lie T-duality transformations, we have obtained the solution of the equations of motion of the dual sigma model. Finally, we were able to find an example of the super non-Abelian T-dual conformal sigma models (at least at the one-loop level) for which the vanishing $\beta$-function equations are satisfied. By solving the vanishing $\beta$-function equations we found the general form of the dilaton field of the models and showed that the dilaton field of the original model is invariant under the flat supercoordinates transformation; that is, one can go from $\varphi(\Phi)$ to $\bar{\varphi}(\Omega)$ and vice versa.

\section{Acknowledgments}

The author would like to thank the anonymous referee for invaluable comments and criticisms. Also, the author is grateful to A. Rezaei-Aghdam for his valuable comments and H. Shams for carefully reading the manuscript.

Open Access. This article is distributed under the terms of the Creative Commons Attribution License (CC-BY 4.0), which permits any use, distribution and reproduction in any medium, provided the original author(s) and source are credited.

\section{References}

[1] M. Henneaux and L. Mezincescu, A $\sigma$-model interpretation of Green-Schwarz covariant superstring action, Phys. Lett. B 152 (1985) 340 [INSPIRE].

[2] N. Read and H. Saleur, Exact spectra of conformal supersymmetric nonlinear $\sigma$-models in two-dimensions, Nucl. Phys. B 613 (2001) 409 [hep-th/0106124] [INSPIRE].

[3] S. Guruswamy, A. LeClair and A.W.W. Ludwig, $g l(N \mid N)$ super-current algebras for disordered Dirac fermions in two-dimensions, Nucl. Phys. B 583 (2000) 475 [cond-mat/9909143] [INSPIRE].

[4] R.R. Metsaev and A.A. Tseytlin, Type IIB superstring action in $A d S_{5} \times S^{5}$ background, Nucl. Phys. B 533 (1998) 109 [hep-th/9805028] [INSPIRE].

[5] N. Berkovits, C. Vafa and E. Witten, Conformal field theory of AdS background with Ramond-Ramond flux, JHEP 03 (1999) 018 [hep-th/9902098] [INSPIRE].

[6] M. Bershadsky, S. Zhukov and A. Vaintrob, $\operatorname{PSL}(n \mid n) \sigma$-model as a conformal field theory, Nucl. Phys. B 559 (1999) 205 [hep-th/9902180] [INSPIRE].

[7] N. Berkovits, M. Bershadsky, T. Hauer, S. Zhukov and B. Zwiebach, Superstring theory on $A d S_{2} \times S^{2}$ as a coset supermanifold, Nucl. Phys. B 567 (2000) 61 [hep-th/9907200] [INSPIRE].

[8] J. de Boer and S.L. Shatashvili, Two-dimensional conformal field theories on AdS $(2 d+1)$ backgrounds, JHEP 06 (1999) 013 [hep-th/9905032] [INSPIRE].

[9] D. Kagan and C.A.S. Young, Conformal $\sigma$-models on supercoset targets, Nucl. Phys. B 745 (2006) 109 [hep-th/0512250] [INSPIRE].

[10] T.H. Buscher, A symmetry of the string background field equations, Phys. Lett. B 194 (1987) 59 [InSPIRE]. 
[11] T.H. Buscher, Path integral derivation of quantum duality in nonlinear $\sigma$-models, Phys. Lett. B 201 (1988) 466 [INSPIRE].

[12] M.J. Duff, Duality rotations in string theory, Nucl. Phys. B 335 (1990) 610 [InSPIRE].

[13] A. Giveon and M. Roček, Generalized duality in curved string backgrounds, Nucl. Phys. B 380 (1992) 128 [hep-th/9112070] [INSPIRE].

[14] E. Kiritsis, Exact duality symmetries in CFT and string theory, Nucl. Phys. B 405 (1993) 109 [hep-th/9302033] [INSPIRE].

[15] M. Roček and E.P. Verlinde, Duality, quotients and currents, Nucl. Phys. B 373 (1992) 630 [hep-th/9110053] [INSPIRE].

[16] X.C. de la Ossa and F. Quevedo, Duality symmetries from non-Abelian isometries in string theory, Nucl. Phys. B 403 (1993) 377 [hep-th/9210021] [INSPIRE].

[17] C. Klimčík and P. Ševera, Dual non-Abelian duality and the Drinfeld double, Phys. Lett. B 351 (1995) 455 [hep-th/9502122] [INSPIRE].

[18] V.G. Drinfeld, Quantum groups, in the Proceedings of the International Congress of Mathematicians, Berkeley U.S.A. (1986), American Mathematical Society, New York U.S.A. (1987), pg. 798.

[19] L. Hlavaty, Classical solution of a $\sigma$-model in curved background, Phys. Lett. B 625 (2005) 285 [hep-th/0506188] [INSPIRE].

[20] L. Hlavaty, J. Hybl and M. Turek, Classical solutions of $\sigma$-models in curved backgrounds by the Poisson-Lie T-plurality, Int. J. Mod. Phys. A 22 (2007) 1039 [hep-th/0608069] [INSPIRE].

[21] L. Hlavatý and L. Šnobl, Poisson-Lie T-plurality of three-dimensional conformally invariant $\sigma$-models. II. Nondiagonal metrics and dilaton puzzle, JHEP 10 (2004) 045 [hep-th/0408126] [INSPIRE].

[22] L. Hlavatý and M. Turek, Flat coordinates and dilaton fields for three-dimensional conformal sigma models, JHEP 06 (2006) 003 [hep-th/0512082] [INSPIRE].

[23] L. Hlavatý, Dilatons in curved backgrounds by the Poisson-Lie transformation, hep-th/0601172 [INSPIRE].

[24] A. Eghbali and A. Rezaei-Aghdam, Poisson-Lie T-dual $\sigma$-models on supermanifolds, JHEP 09 (2009) 094 [arXiv:0901.1592] [INSPIRE].

[25] A. Eghbali and A. Rezaei-Aghdam, String cosmology from Poisson-Lie T-dual $\sigma$-models on supermanifolds, JHEP 01 (2012) 151 [arXiv:1107.2041] [INSPIRE].

[26] A. Eghbali and A. Rezaei-Aghdam, WZW models as mutual super Poisson-Lie T-dual $\sigma$-models, JHEP 07 (2013) 134 [arXiv: 1303.4069] [INSPIRE].

[27] A. Eghbali and A. Rezaei-Aghdam, Super Poisson-Lie symmetry of the GL(1|1) WZNW model and worldsheet boundary conditions, Nucl. Phys. B 866 (2013) 26 [arXiv:1207.2304] [INSPIRE].

[28] N. Backhouse, A classification of four-dimensional Lie superalgebras, J. Math. Phys. 19 (1978) 2400 [INSPIRE].

[29] B. DeWitt, Supermanifolds, Cambridge University Press, Cambridge U.K. (1992). 
[30] A. Eghbali, A. Rezaei-Aghdam and F. Heidarpour, Classification of four and six dimensional Drinfel'd superdoubles, J. Math. Phys. 51 (2010) 103503 [arXiv:0911.1760] [InSPIRE].

[31] A. Eghbali, A. Rezaei-Aghdam and F. Heidarpour, Classification of two and three dimensional Lie super-bialgebras, J. Math. Phys. 51 (2010) 073503 [arXiv:0901.4471] [INSPIRE].

[32] C. Klimčík and P. Ševera, Poisson-Lie T duality and loop groups of Drinfeld doubles, Phys. Lett. B 372 (1996) 65 [hep-th/9512040] [INSPIRE].

[33] C. Klimčík and P. Ševera, Poisson Lie T duality: open strings and D-branes, Phys. Lett. B 376 (1996) 82 [hep-th/9512124] [INSPIRE].

[34] T. Tokunaga, String theories on flat supermanifolds, Report Number YITP-05-47, [hep-th/0509198] [INSPIRE]. 